\title{
COMMENTARY
}

\section{Burns: learning from the past in order to be fit for the future}

\author{
Lars-Peter Kamolz* \\ See related research by Williams et al., http://ccforum.com/content/13/6/R183
}

\begin{abstract}
Many advances have been made in the understanding and treatment of burns. Advances in burn surgery and critical care have decreased mortality and morbidity. Survival from severe burns is no longer the exception, but unfortunately death still occurs. Williams and colleagues have determined in their recent paper the predominant causes of death in order to develop new treatment avenues and future trajectories suitable to increase survival and overall outcome. A lot of burn deaths may be preventable with better airway management and a more precise and adequate volume management, but the leading cause of death in patients suffering from severe burns, which has to be faced, is sepsis. Sepsis due to multidrug-resistant organisms will continue to impede efforts to increase survival, and new strategies that go beyond the surgical and clinical techniques, which are already implemented, have to be developed in order to fight these organisms and their related complications.
\end{abstract}

In the previous issue of Critical Care, Williams and colleagues [1] provide an overview of the predominant causes of death in burned pediatric patients in order to develop new treatment avenues and future trajectories.

Over the past decades there has been a significant decrease in mortality and morbidity in severe burns due to improved burn wound management and approaches in critical care [2-4]. Many advances have been made, not only concerning the pathophysiology of burns [5] but also concerning burn management. Survival from severe burns is no longer the exception, but unfortunately death still occurs [1].

\footnotetext{
${ }^{*}$ Correspondence: lars-peter.kamolz@meduniwien.ac.at

Vienna Burn Center, Division of Plastic and Reconstructive Surgery, Department of Surgery, Medical University of Vienna, Waehringer Guertel 18-20, 1090 Vienna, Austria
}

Owing to the fact that the burn injury is multifaceted, the advances cross many injury processes. These issues range from the management of the catabolic state [6] to modern wound care. One important aspect is that burn treatment has become more proactive, by searching out new technologies to solve old problems. Now the treatment approach is altering its focus on manipulating the course of a burn and its final outcome. The survival rate is still of course the most important issue, but not the only issue [7]. The question of whether and to what degree the patient is able to enjoy a normal quality of life becomes more and more essential in how the outcome of the burn treatment is evaluated [7]. Restoring function and esthetics are crucial in the difficult process of social reintegration and the return to a normal life. Great efforts were made in the past to develop epidermal and dermal replacements to overcome the problem of poor skin quality and scar contraction. In large and deep burns, the approach has changed to rapid excision [1] and lesionspecific coverage of the burn wound, eliminating the burn as a source of complications. Rapid and effective wound coverage and closure are of utmost importance, but infection control and the preservation of active and passive motion are also essential for optimal recovery.

Nonviable burn tissue is well recognized to be the driving force behind wound infection and burn wound sepsis. Infection in burn patients remains the significant source of morbidity and mortality. Williams and colleagues, who determined the predominant causes of death of burned children admitted between 1989 and 2009, found that the dominant cause of death is sepsis (47\% of all deaths) [1]. Moreover, they found an increase of deaths due to multidrug-resistant organisms from $42 \%$ to $86 \%$ over the past 20 years. The aggressive use of antimicrobials has significantly improved survival, but has also led to an increased colonization of pathogens that have resistance to current therapies. In general, early removal and excision of the necrotic tissue with a consecutive rapid and effective closure of the burn wound has become the standard in the management of severe burns. Research has proven that application of 
antimicrobial dressings or early excision and grafting is the key to avoid burn wound infection and its extension to systemic infection [8-10]. The timing and extent of surgery may vary, as well as the method of closing, between different burn centers, but the principal concept is almost the same.

Based on the findings of Williams and colleagues, respiratory failure accounted for $29 \%$ of all deaths $-83 \%$ of these were due to acute respiratory distress syndrome [1]. Although the methods used for the management of acute respiratory distress syndrome have changed dramatically over the past 20 years, mortality has remained almost the same $[1,11]$. These observations highlight the need for effective intervention methods for this highly lethal syndrome. Moreover, it seems that there is a need for further studies or for a revisit to the manner in which studies are conducted and their results are implemented in the real world [11].

Resuscitation is the major component of initial burn care and must be managed to restore and preserve organ function. Prevention of inadequate perfusion, due to burn fluid loss, remains the top priority for initial management. Advances in fluid management have led to a marked decrease in fatal burn shock and its related complications. Williams and colleagues reported that shock accounted for $8 \%$ of their deaths [1]. The obvious challenge concerning resuscitation is to provide enough fluid to maintain perfusion without causing overload $[3,12,13]$. Without effective and rapid intervention, hypovolemia will develop. A delay in fluid resuscitation beyond 2 hours of the burn injury complicates resuscitation and increases mortality [14]. The consequences of excessive resuscitation and fluid overload are as deleterious as those of under-resuscitation: pulmonary edema, myocardial edema, conversion of superficial into deep burns, the need for fasciotomies and abdominal compartment syndrome. A recent approach has led to conversion of a formula-driven process to a more critical care approach using more physiologic endpoints such as urinary output and other measurements, so the trend in burn resuscitation is shifting the focus from fluid formulas to adequate endpoint monitoring, edema control and adjuvant therapies $[12,15,16]$.

On some level, a lot of burn deaths may be preventable with better airway management and more precise and adequate volume management. Sepsis due to multidrugresistant organisms, however, will continue to impede efforts to increase survival. We have to develop strategies to fight these organisms that go beyond the surgical and clinical techniques that are already implemented. Moreover there will be a need for further studies that are facing the problems concerning respiratory and multiorgan failure.

Competing interests

The author declares that he has no competing interests.

Published: 10 February 2010

\section{References}

1. Williams FN, Herndon DN, Hawkins HK, Lee JO, Cox RA, Kulp GA, Finnerty CC, Chinkes DL, Jeschke MG: The leading causes of death after burn injury in a single pediatric burn center. Crit Care 2009, 13:R183.

2. Demling $\mathrm{RH}$ : Burns: what are the pharmacological treatment options? Expert Opin Pharmacother 2008, 9:1895-1908.

3. Latenser BA: Critical care of the burn patient: the first 48 hours. Crit Care Med 2009, 37:2819-2826.

4. Pruitt BA, Wolf SE: An historical perspective on advances in burn care over the past 100 years. Clin Plastic Surg 2009, 36:527-545.

5. Keck M, Herndon DN, Kamolz LP, Frey M, Jeschke MG: Pathophysiology of burns. Wien Med Wochenschr 2009, 159:327-336.

6. Williams FN, Herndon DN, Jeschke MG: The hypermetabolic response to burn injury and interventions to modify this response. Clin Plast Surg 2009, 36:583-596.

7. Haslik W, Kamolz LP, Nathschläger G, Andel H, Meissl G, Frey M: First experiences with the collagen-elastin matrix Matriderm as a dermal substitute in severe burn injuries of the hand. Burns 2007, 33:364-368.

8. Polavarapu N, Ogilvie MP, Panthaki ZJ: Microbiology of burn wound infections. J Craniofac Surg 2008, 19:899-902.

9. Patel PP, Vasquez SA, Granick Ms, Rhee ST: Topical antimicrobials in pediatric burn wound management. J Craniofac Surg 2008, 19:913-922.

10. Church D, Elsayed S, Reid O, Winston, Lindsay R: Burn wound infections. Clin Microbiol Rev 2006, 19:403-434.

11. Phua J, Badia JR, Adhikari NJ, Friedrich JO, Fowler RA, Singh JM, Scales DC, Stather DR, Li A, Jones A, Gattas DJ, Hallett D, Tomlinson G, Stewart TE, Ferguson ND: Has mortality from acute respiratory distress syndrome decreased over time? Am J Respir Crit Care Med 2009, 179:220-227.

12. Tricklebank S: Modern trends in fluid therapy for burns. Burns 2009, 35:757-767.

13. Dulhunty JM, Boots RJ, Rudd MJ, Muller MJ, Lipmann J: Increased fluid resuscitation can lead to adverse outcomes in major-burn injured patients, put low mortality is achievable. Burns 2008, 34:1090-1097.

14. Barrow RE, Jschke MG, Herndon DN: Early fluid resuscitation improves outcomes in severely burned children. Resuscitation 2000, 45:91-96.

15. Dries DJ: Management of burn injuries - recent developments in resuscitation, infection control and outcomes research. Scand J Trauma Resusc Emerg Med 2009, 17:14-27.

16. Ahrns KS: Trends in burn resuscitation: shifting the focus from fluids to adequate endpoint monitoring, edema control, and adjuvant therapies. Crit Care Nurs Clin North Am 2004, 16:75-98.

doi:10.1186/cc8192

Cite this article as: Kamolz L-P: Burns: learning from the past in order to be fit for the future. Critical Care 2010, 14:106. 